\title{
Review
}

\section{How we deal with Staphylococcus aureus (MSSA, MRSA) central nervous system infections}

\author{
Roberta Maria Antonello ${ }^{1}$, Niccolò Riccardi ${ }^{2}$ * \\ ${ }^{1}$ Azienda Sanitaria Universitaria Giuliano Isontina ASUGI, Trieste University Hospital, 34149 Trieste, Italy \\ ${ }^{2}$ Infectious Diseases Unit, Department of Clinical and Experimental Medicine, University of Pisa, 56124 Pisa, Italy \\ *Correspondence: niccolo.riccardi@yahoo.it (Niccolò Riccardi) \\ Academic Editor: Gustavo Caetano-Anollés \\ Submitted: 21 November 2021 Revised: 18 December 2021 Accepted: 23 December 2021 Published: 12 January 2022
}

\begin{abstract}
Among central nervous system (CNS) infections (e.g., meningitis, brain abscess, ventriculitis, transverse myelitis), those caused by Staphylococcus aureus (SA) are particularly challenging both in management and treatment, with poor clinical outcomes and long hospital stay. It has been estimated that SA is responsible for around $1 \%-7 \%$ of meningitis (up to $19 \%$ in healthcare-associated meningitis). Recent neurosurgical procedures and immunocompromisation are major risk factors for SA CNS infections. Hand hygiene, surveillance nasal swabs and perioperative prophylaxis are crucial points for effective SA infections prevention. In case of SA-CNS infections, pending microbiological results, anti-methicillin-resistant SA (MRSA) antibiotic, with good CNS penetration, should be included, with prompt de-escalation as soon as MRSA is ruled out. Consultation with an expert in antimicrobial therapy is recommended as well as prompt source control when feasible. In this narrative review, we reviewed current literature to provide practical suggestions on diagnosis, prevention, management, and treatment of SA CNS infections.
\end{abstract}

Keywords: Staphylococcus aureus; Central nervous system; CNS; MSSA; MRSA; Management; Review

\section{Introduction}

Bacterial central nervous system (CNS) infections are serious infections requiring prompt evaluation and management [1]. Etiological causes of bacterial meningitis vary by group age; in fact, among neonates and children most cases are due to group B Streptococcus agalactiae, Escherichia coli, Listeria monocytogenes, Haemophilus influenzae, and Streptococcus pneumoniae. Among adults, Streptococcus pneumoniae, Neisseria meningitidis, and Listeria monocytogenes represents the most detected cause of community acquired bacterial meningitis. Staphylococcus aureus (SA) CNS infection with its multiple clinical presentations (e.g., meningitis, brain abscess) is a rare but life-threatening condition, often occurring post neurosurgical interventions and/or in immunocompromised patients $[1,2]$.

Treatment and management are challenging because of the critical location and the reduced penetration of systemically administered antibiotics in the CNS due to the blood-brain barrier. In addition, in case of methicillinresistant SA (MRSA), a worse outcome may occur due to limited therapeutic options and pathogen related factors, such as adhesin genes, immune evasion genes, hemolysin and toxins (e.g., pore-forming toxins, Panton-Valentine leukocidin toxin) $[3,4]$. Furthermore, SA easily forms biofilm, especially in case of foreign bodies, requiring therapy with anti-biofilm properties or, in some cases, combination of two effective anti-SA agents [5]. It is estimated that MRSA accounts for about one third of SA CNS infections, thus raising attention on the importance of an appropriate reasoned empiric antibiotic treatment pending antimicrobial susceptibility test results [2].

Hand hygiene, surveillance swabs and adequate perioperative prophylaxis are crucial, as well as antimicrobial stewardship programs, to limit morbidity and costs related to prolonged hospital stay [6].

Aim of this narrative review is to raise attention on SA CNS infections, offering clinicians practical tips on diagnosis, prevention, antibiotic treatment and management.

\section{Methods}

On October 25th 2021 we performed a PubMed/MEDLINE literature search. The complete search string was " $(((()$ central nervous system infection[Title/Abstract]) OR (CNS infection[Title/Abstract])) OR ((brain abscess[Title/Abstract]) OR (cerebral abscess[Title/Abstract]))) OR (encephalitis[Title/Abstract])) OR (meningitis[Title/Abstract])) OR (myelitis[Title/Abstract])) OR (spinal cord[Title/Abstract])) AND (aureus[Title/Abstract])". 1350 papers were found. Of these, 245 were excluded because they were not written in English and 895 were excluded by title or abstract screening because they were not pertinent with the review's topic. Two hundred and ten papers were screened by full text and discussed by the Authors, who jointly made the final decision about which papers to consider for inclusion. Seventy-four papers were included in the present review. Their pertinent references were evaluated and eventually included in the manuscript. 
Table 1. Principal risk factors and diagnostic tools for $S$. aureus central nervous system infections.

\begin{tabular}{ll}
\hline Risk factors & Diagnostic tools \\
\hline Recent surgery & Physical examination \\
SA bloodstream infection & Blood examination \\
SA infection (e.g., infective endocarditis, pneumonia, aortic graft infection) & CSF examination \\
Infectious of contiguous site (e.g., otitis) & CSF serology \\
Presence of foreign bodies & PCR on CSF or intraoperatory specimens \\
MRSA colonization & Cultures (CSF, blood, pus) \\
Immunocompromisation & Neuroradiology \\
Diabetes mellitus & Nuclear medicine \\
Cancer & - \\
Dialysis & - \\
\hline SA, Staphylocus
\end{tabular}

SA, Staphylococcus aureus; MRSA, methicillin-resistant S. aureus; CSF, cerebrospinal fluid; PCR, polymerase chain reaction.

The manuscript was organized in the following major chapters: "Epidemiology and risk factors"; "Clinical presentation"; "Diagnosis"; "Prevention and prophylaxis"; "Reasoned antibiotic treatment and management".

\section{Epidemiology and risk factors}

\subsection{Epidemiology}

SA CNS infections incidence greatly depends on the setting and the geographical region of investigation. For instance, the number of meningitis that are caused by SA is around four times higher in Europe than in Africa or America (different microbiological detection methods and publication bias should always be considered) [7]. 1\%-7\% of meningitis are reported to be caused by SA, with up to $19 \%$ in healthcare associated meningitis and up to $41 \%$ of brain abscesses [7-10]. In children, SA is reported to cause up to $32 \%$ of acute CNS infections [11,12].

\subsection{Risk factors}

SA can infect the CNS through three main paths: contiguous spread from nearby body sites, haematogenous dissemination (including septic vegetations on heart valves), and during surgical intervention (Table 1). Surgical interventions represent a risk factor both in case of direct involvement of the CNS and in case of ophthalmic or otolaryngologic procedures [13]. In post-operative CNS infections, cerebrospinal fluid (CSF) leak and external ventricular drainage are adjunctive risk factors [14]. Emergency surgical procedures expose the patient to a greater risk of SA infection compared to elective surgery [15].

Immune deficits, both innate and acquired, are known risk factors for CNS infections sustained by uncommon pathogens, including Gram positive bacteria [16]. Despite this, CNS infections sustained by SA have been described also in immunocompetent patients without any known risk factors [17]. Furthermore, immunomodulation occurring in pregnancy has been occasionally reported as a risk factor for the spreading of SA to CNS from contiguous sites [18].
Diabetes mellitus, cancer and dialysis are reported to be adjunctive risk factors as well as negative prognostic factors [19,20]. Foreign bodies are a risk factor for bacterial infection, both in case of neurosurgical devices (e.g., deep brain stimulator), but also in case of central venous catheters, and dialysis equipment [21,22]. Of note, patients carrying a ventriculoperitoneal shunt should always be considered at higher risk of intra-abdominal bacterial migration to CNS and SA is occasionally reported as the causative agent of CNS infections also in this peculiar scenario [23]. Mariano et al. [24] described the case of a young woman with MRSA endocarditis and meningitis occurring after a nape piercing removal, underlying the importance of careful local hygiene after any invasive procedures.

In addition, neurologic complications of infective endocarditis should be kept in mind. Patients suffering from SA infective endocarditis may experience bacterial dissemination to the CNS, as SA vegetations are typically large and friable and therefore with a high embolic potential [25]. Among endocarditis, those caused by SA are reported to account as an independent risk factor for neurologic complications [26]. Cardiac vegetations causing septic emboli to CNS, microabscesses and mycotic aneurysms, potentially leading to ischemic or hemorrhagic stroke, are described in literature [27]. Risk factors for endocarditis and SA bacteremia (e.g., intravenous drug abuse), are risk factors for SA CNS infections as well [27].

Finally, special attention is required for peculiar settings: due to the limited diagnostic options, delay in prompt diagnosis and treatment of MRSA CNS infections in prisons and shelters, as well as in long-term facilities, is associated with death or severe sequelae $[6,28]$.

\section{Clinical presentation}

Clinical presentation depends on the exact localization of infection (e.g., encephalitis, brain abscess, empyema, meningitis). Despite this, common abnormalities on physical examination are present in most cases: fever with 
chills, tachycardia, hypotension and general malaise are commonly found [29]. However, these signs may also be absent and the patient can be hemodynamically stable [30]. The mental status may be altered and the Glasgow Coma Scale (GCS) score may be low [14]. Signs of meningeal irritation (e.g., Brudzinski sign, Lasegue sign) are commonly present in case of meningeal involvement, as well as vomit and nuchal rigidity [31]. Other neurological symptoms, such as seizures, hallucinations, confusion, vision loss, and neurological deficits should alway be carefully evaluated [31].

Meningitis is a medical emergency requiring immediate antibiotic prescription and supportive therapy. SA meningitis is reported in most cases as post neurosurgical complication, but due to anatomical proximity, meningitis may occur as a threatening complication of ocular/orbital infections and otitis [32]. It has also been described as a complication of a furuncle whose cultures grew MRSA [33]. Spontaneous MRSA meningitis in an otherwise healthy patient has also been described [31,34]. Cerebrovascular complications of meningitis (e.g., intracranial haemorrhage, cerebral infarction, cavernous sinus thrombosis) are rarely described $[35,36]$. Spinal epidural phlegmon and in general spinal cord involvement have occasionally been reported and are associated with poor outcomes [30,37]. Pneumocephalus, defined as the presence of gas in the cranial cavity, is another extremely rare complication of SA meningitis [38]. Meningitis and brain abscesses can occasionally be present simultaneously, also without known predisposing factors [39].

Abscesses are focal suppurative processes that can be parenchymal, subdural or in continuity with adjacent structures [40] (Fig. 1). Post neurosurgical brain abscesses can occur weeks to months after surgical intervention (up to two years later) [9]. Intraventricular extension is associated with high mortality rates (up to $80 \%$ ), which are doubled compared to abscesses non involving ventricular space [41]. Multifocal brain abscesses as a complication of endophthalmitis following ophthalmic procedures or chorioretinitis were reported also in immunocompetent patients [17,29]. Gan et al. [42] described a brain abscess caused by MRSA occurring in an otherwise healthy infant as a conjunctivitis complication. A patient suffering from brain abscess should always be investigated for immunodeficiency, and in case of infants also for specific congenital immune deficits [43]. Epidural spinal abscesses can compress the spinal cord and nerve roots, resulting in motor and sensory lateralized deficits. Okada et al. [44] described the case of a middle-age man with an epidural abscess involving the entire spine who had undergone sacral epidural block two weeks before presentation, presenting with signs of meningeal irritation but no neurological deficits.

Pyogenic ventriculitis consists of suppurative fluid in the cerebral ventricles and it can occur both as a post neurosurgical complication, especially in external ventricular

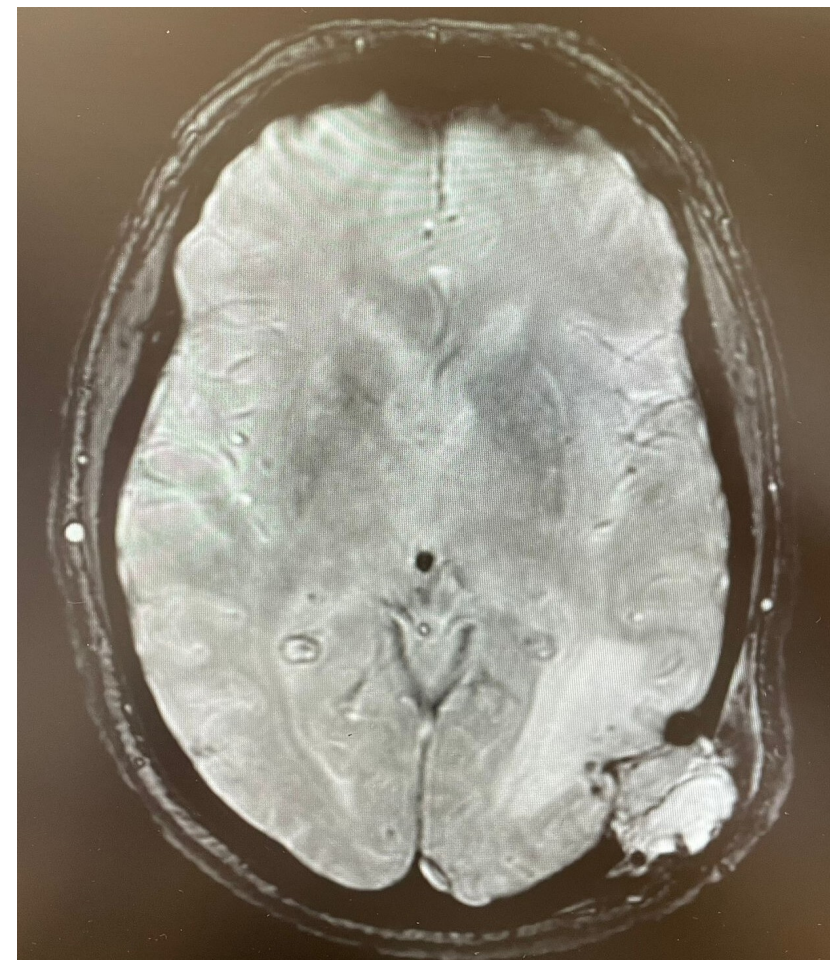

Fig. 1. MRSA abscess occurring after left occipital craniotomy. MRI sequences show a mixed-content (purulent and transudative/phlogistic) harvest in correspondence with the surgical site. The purulent extracranial compartment displaces the contiguous soft tissue ("mass effect") and it is interconnected with satellite infectious lesions in the parietal parasagittal posterior region. Meningeal enhancement and vasogenic edema in the left occipital region are also described.

drains carrier, or as primary pyogenic ventriculitis complicating community-acquired meningitis [45]. Primary ventriculitis is an extremely rare condition, with less than 10 cases described. Signs and symptoms are extremely variable and signs of meningeal irritation and focal neurological deficits are often absent [46,47].

SA acute transverse myelitis is a rare condition with few cases reported and it is often associated with poor outcomes $[48,49]$. It usually occurs soon after an infection involving another site, suggesting an immune-mediated effect $[50,51]$. Neurological deficits are often present and osteomyelitis can occur simultaneously [48].

Mild encephalitis/encephalopathy with reversible splenial lesions (MERS) is a rare clinico-radiological syndrome first described in 2004 and occasionally reported due to SA bacteraemia [52].

\section{Diagnosis}

\subsection{Physical examination}

Medical history should always be assessed carefully, paying particular attention to the immune system status, recent surgery, other signs and symptoms that may suggest 
the site of origin of infection. Physical examination should also exclude a concomitant infective endocarditis, sinusitis, otitis, mastoiditis, and oral cavity poor hygiene suggestive of odontogenic foci [42,53]. Previous MRSA colonization should also be asked.

\subsection{Blood examination}

Blood tests usually show elevated inflammation markers, with leukocytosis (most neutrophils) and elevated $\mathrm{C}$ reactive protein (CRP) and procalcitonin levels $(>2 \mathrm{ng} / \mathrm{mL})$ $[10,44]$. Blood cultures may be of support in identifying the causative agent, although they turn positive only in about one third of cases [54].

\subsection{Lumbar puncture}

Lumbar puncture is crucial for the diagnosis of CNS infections. In case of SA meningitis, CSF examination commonly reveals high levels of white blood cells (most neutrophils), elevated protein level and hypoglycorrhachia $[31,44]$. Normal CSF glucose levels are occasionally reported $[33,55]$. Of note, presentation of CSF in SA meningitis is common to that of other bacterial meningitis. This should always be kept in mind not to miss differential diagnosis pending microbiological results. CSF examination can result within normal ranges, especially in case of brain abscesses [14]. CSF Gram stain and cultures identify the causative agent in most cases [31], allowing antimicrobial susceptibility testing and targeted antibiotic therapy. Serology on CSF and antigen testing can raise suspicion on the causative agent pending culture results, and can rapidly distinguish, besides CSF examination, between bacterial, viral or fungal CNS infections. Polymerase chain reaction (PCR) and its variants (e.g., multiplex PCR, microarray assay) performed on significant samples (e.g., CSF, drained pus) is a valid rapid diagnostic option with good specificity and sensitivity [56,57]. PCR can also distinguish between MSSA and MRSA using specific primers for nuclease gene and $\mathrm{Mec}$ gene, respectively [56]. In case of drainage of an abscess, pus cultures may lead to the diagnosis in case of inconclusive lumbar puncture test or blood culture results.

\subsection{Neuroradiology}

Imaging investigation is of support on clinical suspicion, with specific features according to the localization of infection. Computed tomography (CT) has a good sensitivity in detecting brain abscesses, usually presenting with a thin, regular enhancing capsule, but it has some problems in making differential diagnosis (e.g., brain tumours) [41]. With regards to meningitis or ventriculitis, CT scan is often normal and the imaging evaluation requires magnetic resonance imaging (MRI) sequences [45]. Of note, also standard MRI sequences may result inadequate in diagnosing CNS infections [45]. On the other hand, diffusionweighted MRI sequences have a higher sensitivity in diagnosing ventriculitis and meningitis [45]. In case of meningi- tis, diffusion-weighted MRI often shows multiple subarachnoid hyperintense lesions, mainly distributed in the frontal lobe region [58]. Diffusion-weighted MRI is able to differentiate brain abscesses from neoplasia in most cases, but differential diagnosis remains challenging [59]. Magnetic resonance spectroscopy is reported to discriminate also between aerobic and anaerobic brain abscesses [60]. Differential diagnosis of brain abscesses includes brain tumours and neuroinflammation, therefore an accurate diagnosis at an early stage is fundamental to define the best approach [41]. As an example, Pham et al. [61] reported the extremely rare condition of an infected intradural dermoid cyst mimicking a brain abscess on MRI. Imaging techniques are sometimes employed also for etiological diagnosis and source control purposes, as in the case of CT- or MRI-guided biopsies or drainage [62].

\subsection{Nuclear medicine}

Nuclear medicine is gaining ground in recent years on CNS infections diagnosis. Photon emission tomography CT (PET CT) is highly sensitive in detecting meningeal enhancement [22]. CNS evaluation is challenging because the brain is a high glucose metaboliser also under physiological conditions, therefore the best radiopharmaceutical should be carefully evaluated.

\section{Prevention and prophylaxis}

It is estimated that $30-50 \%$ of adults are colonized with MRSA though totally asymptomatic, therefore screening programs (e.g., nasal swabs, skin swabs) are crucial to identify patients at higher risk of MRSA invasive infection $[6,63]$. As most MRSA invasive infections occur in patients without a history of MRSA colonization, besides in patients being colonized with MRSA, prophylaxis for SA should be carefully evaluated in every immunocompromised patient undergoing neurosurgery $[64,65]$. Time is a critical issue: screening should be performed as close to the operation date as possible, but in time to perform decolonization and/or preoperative prophylaxis. Optimal preoperative prophylaxis should be administered 30 to $60 \mathrm{~min}$ before the incision and prophylactic antimicrobial agents should be discontinued within $24 \mathrm{~h}$ of the procedure [6]. Due to organizational issues, pre-procedural screening is sometimes impossible to be performed in emergency interventional procedures, thus partially explaining the higher mortality rates in these patients $[15,20]$. With this in mind, high-risk patients undergoing elective surgical procedures should be screened at regular intervals. In case of MRSA colonisation, decolonisation aims at reducing MRSA at sub-detection levels. The best prophylactic regimen (topical, systemic or combination regimen) should be evaluated according to the procedure the patient is going to attend [6]. Short and ultra-short prophylaxis regimens are encouraged. First-generation cephalosporins (e.g., cefazolin) are usually administered $30 \mathrm{~min}$ before surgery if surveillance 


\section{S. aureus CNS infection: reasoned empiric treatment}

Broad-spectrum empiric treatment can be started pending microbiological results, with prompt de-escalation as soon as MRSA is excluded

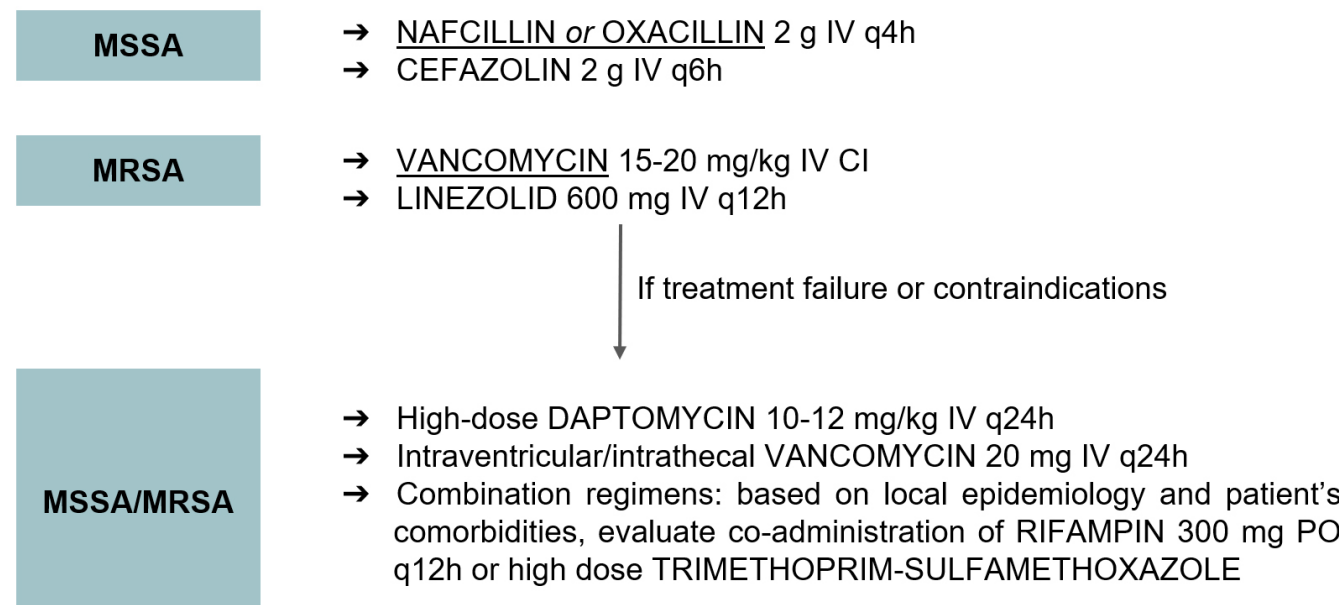

Fig. 2. Staphylococcus aureus central nervous system infections: diagnosis and reasoned empiric antibiotic therapy. First-choice treatments are underlined. Drug dosages refer to adults without renal or hepatic impairment. The choice of treatment should always take into consideration allergies and comorbidities of the patient. Advice from an expert in antimicrobial therapy is recommended. CI, continuous infusion; CSF, cerebrospinal fluid; CNS, central nervous system; IV, intravenously; MSSA, methicillin susceptible $S$. aureus; MRSA, methicillin susceptible S. aureus; PCR, polymerase chain reaction; PO, orally; SA, Staphylococcus aureus.

nasal swabs did not detect MRSA. On the contrary, vancomycin is preferred in case of known MRSA colonization or $\beta$-lactam allergy [66]. If hair removal is necessary, razor blade use is discouraged and electric clipping or depilatory cream should be used instead [67]. Antimicrobial stewardship programs regarding both preoperative and postoperative prophylaxis based on local epidemiology are advisable.

Beside surgical prophylaxis, general behaviours to reduce MRSA spreading should be adopted in both medical and surgical wards. Hand washing is the most effective preventive measure [68]. In addition, patients' belongings should be personal and not shared, waste and laundry appropriately managed, and surfaces should be periodically cleaned [6].

\section{Reasoned antibiotic treatment}

Taking in account patients' comorbidities (e.g., chronic renal failure) and possible drug-drug interactions (DDIs), empiric treatment should cover MRSA with antibiotics with good CNS penetration and, as soon as MRSA has been ruled out, prompt de-escalation to anti-MSSA treatment should be carried out (Fig. 2).

First line antibiotics for MRSA CNS infections are vancomycin $15-20 \mathrm{mg} / \mathrm{kg}$ intravascular (IV) continuous infusion, with dose adjustment according to renal function, to achieve preferred target AUC24 of $400-600 \mu \mathrm{g} / \mathrm{mL}$ or trough concentration of $15-20 \mu \mathrm{g} / \mathrm{mL}$ and linezolid $600 \mathrm{mg}$ every $12 \mathrm{~h}$ (always paying attention to possible DDIs) [6971]. Based on antimicrobial susceptibility test (AST), deescalation to nafcillin or oxacillin $2 \mathrm{~g}$ IV every $4 \mathrm{~h}$ represents a good anti-MSSA option [72]. In a recent systematic review, cefazolin was found to be non inferior to antistaphylococcal penicillins in treating MSSA bacteremia and it was associated with inferior nephrotoxicity: due to good CNS penetration, cefazolin may be considered as an alternative option for MSSA CNS infections [72,73]. Limited data are available about ceftaroline, a fifth generation cephalosporin with anti-MSSA/MRSA activity, in S. aureus CNS infections; however, in animal models and few human case reports, a good CNS penetration and treatment outcome have been reported [74,75].

In a recent randomized control trial, high dose trimethoprim-sulfamethoxazole did not achieve noninferiority to vancomycin in the treatment of severe MRSA infections and its use is only limited as companion drug to anti-MSSA/MRSA first-line antibiotics [76].

High-dose daptomycin (10-12 mg/Kg) may be considered as salvage therapy for MSSA/MRSA CNS infection in patients that have failed or have contraindication to first line agents [77].

Combination therapy with two active agents (e.g., rifampin $300 \mathrm{mg}$ every $12 \mathrm{~h}$ or high dose trimethoprimsulfamethoxazole) may be used when no source control can be applied or microbiological/clinical failure of monother- 
apy [78]. Furthermore, intraventricular/lumbar intrathecal administration of vancomycin $20 \mathrm{mg}$ daily may be considered for refractory cases [79]. Whatever the antibiotics chosen, treatment duration should be tailored on patient's clinical, radiological and surgical resolution (treatment lasting less than two weeks are strongly discouraged).

Finally, source control, that is the measures adopted to control the foci of infection (e.g., abscess drainage, removal or substitution of ventriculo-peritoneal shunt) should be applied whenever possible [80].

In case of treatment failure, combined surgical and antibiotic therapy reassessment should be performed to ensure adequate source control.

\section{Conclusions}

SA CNS infections are burdened by high morbidity and mortality. Prompt diagnosis and combined effective source control and antibiotic treatment are mandatory to increase the likelihood of good outcomes.

\section{Author contributions}

RMA-literature search, manuscript draft; NRconception and design, manuscript draft, manuscript revision. All authors read and approved the final manuscript.

\section{Ethics approval and consent to participate}

Not applicable.

\section{Acknowledgment}

Not applicable.

\section{Funding}

This research received no external funding.

\section{Conflict of interest}

The authors declare no conflict of interest.

\section{References}

[1] Erdem H, Inan A, Guven E, Hargreaves S, Larsen L, Shehata G, et al. The burden and epidemiology of community-acquired central nervous system infections: a multinational study. European Journal of Clinical Microbiology \& Infectious Diseases. 2017; 36: 1595-1611.

[2] Pintado V, Pazos R, Jiménez-Mejías ME, Rodríguez-Guardado A, Díaz-Pollán B, Cabellos C, et al. Staphylococcus aureus meningitis in adults: a comparative cohort study of infections caused by meticillin-resistant and meticillin-susceptible strains. The Journal of Hospital Infection. 2019; 102: 108-115.

[3] Cheung GYC, Bae JS, Otto M. Pathogenicity and virulence of Staphylococcus aureus. Virulence. 2021; 12: 547-569.

[4] Dahlberg D, Mariussen E, Goverud IL, Tønjum T, Mæhlen J, Antal E, et al. Staphylococcal $\alpha$-hemolysin is neurotoxic and causes lysis of brain cells in vivo and in vitro. NeuroToxicology. 2015; 48: 61-67.

[5] Al Kandari M, Jamal W, Udo EE, El Sayed A, Al Shammri $\mathrm{S}$, Rotimi VO. A case of community-onset meningitis caused by hospital methicillin-resistant Staphylococcus aureus successfully treated with linezolid and rifampicin. Medical Principles and Practice: International Journal of the Kuwait University, Health Science Centre. 2010; 19: 235-239.

[6] Byrne C, Hazlerigg A, Khan W, Smitham P. The role of perioperative care in reducing rates of methicillin resistant Staphylococcus aureus. Journal of Perioperative Practice. 2011; 21: 410 417.

[7] Oordt-Speets AM, Bolijn R, van Hoorn RC, Bhavsar A, Kyaw MH. Global etiology of bacterial meningitis: a systematic review and meta-analysis. The Public Library of Science one. 2018; 13: e0198772.

[8] Zheng Y, Shang W, Peng H, Rao Y, Zhao X, Hu Z, et al. Virulence Determinants Are Required for Brain Abscess Formation Through Staphylococcus aureus Infection and Are Potential Targets of Antivirulence Factor Therapy. Frontiers in Microbiology. 2019; 10: 682 .

[9] Lange N, Berndt M, Jörger A, Wagner A, Lummel N, Ryang Y, et al. Clinical Characteristics and Course of Postoperative Brain Abscess. World Neurosurgery. 2018; 120: e675-e683.

[10] Sipahi OR, Nazli Zeka A, Taşbakan M, Pullukçu H, Arda B, Yamazhan T, et al. Pooled analysis of 899 nosocomial meningitis episodes from Turkey. Turkish Journal of Medical Sciences. 2018; 47: 29-33.

[11] Gahlot T, Kasana D. A cross-sectional study of etiological and sensitivity profiling of meningitis in under-five children. International Journal of Mycobacteriology. 2021; 10: 149-154.

[12] Iregbu KC, Abdullahi N. Profiles of acute bacterial meningitis isolates in children in National Hospital, Abuja. Nigerian Medical Journal: Journal of the Nigeria Medical Association. 2015; 56: 297-300

[13] Eduwu J, Tabasam F, Bastidas AA, Dar K, Ahmed Y. Successful management of methicillin-resistant Staphylococcus aureusbacteremia complicated with diffuse myelitis. Infectious Diseases. 2017; 49: 234-236.

[14] Zhan R, Zhu Y, Shen Y, Shen J, Tong Y, Yu H, et al. Postoperative central nervous system infections after cranial surgery in China: incidence, causative agents, and risk factors in 1,470 patients. European Journal of Clinical Microbiology \&Amp; Infectious Diseases: Official Publication of the European Society of Clinical Microbiology. 2014; 33: 861-866.

[15] Chen C, Chang C, Lin L, Chen WL, Chang Y, Wang S, et al. Risk factors associated with postcraniotomy meningitis. Medicine. 2016; 95: e4329.

[16] Oliveira APRD, Pappalardo MC, Dantas D, Lins D, Vidal JE. BRAIN ABSCESS DUE to Staphylococcus aureus of CRYPTOGENIC SOURCE in an HIV-1 INFECTED PATIENT in USE of ANTIRETROVIRAL THERAPY. Revista do Instituto De Medicina Tropical De Sao Paulo. 2016; 58: 34.

[17] Mishra A, Giri S, Rauniyar R, Poudel S, Nepal AS, Chaudhary $\mathrm{G}$, et al. Endogenous endophthalmitis and multifocal brain abscess - an interesting case. Clinical Case Reports. 2021; 9: e04913.

[18] Khan S, Siddiqui S. Community-Associated MethicillinResistant Staphylococcus aureus: Case Report of Acute Sinusitis with Orbital Extension in a Pregnant Lady. Cureus. 2020; 12: e12054.

[19] Danyalian A, Padron I, Almeida P, Danckers M. Fulminant methicillin-sensitive Staphylococcus aureus infection: from pinprick to multiorgan failure. British medical journal Case Reports. 2021; 14: e239396.

[20] Wyllie DH, Peto TEA, Crook D. MRSA bacteraemia in patients on arrival in hospital: a cohort study in Oxfordshire 1997-2003. British medical journal. 2005; 331: 992.

[21] Bernstein JE, Kashyap S, Ray K, Ananda A. Infections in Deep Brain Stimulator Surgery. Cureus. 2019; 11: e5440. 
[22] van Rappard JRM, Tolenaar JL, Smits AB, Go PMNYH. Spinal epidural abscess and meningitis following short-term epidural catheterisation for postoperative analgaesia. British medical journal Case Reports. 2015: bcr2015210867.

[23] Marino M, Phillips C. Methicillin-Resistant Staphylococcus aureus Meningitis from Transanal Migration of a Ventriculoperitoneal Shunt. The Journal of Emergency Medicine. 2019; 57: e81-e84.

[24] Mariano A, Pisapia R, Abdeddaim A, Taibi C, Rianda A, Vincenzi $\mathrm{L}$, et al. Endocarditis and meningitis associated to nape piercing in a young female: a case report. Le Infezioni in Medicina. 2015; 23: 275-279.

[25] Cantier M, Mazighi M, Klein I, Desilles JP, Wolff M, Timsit $\mathrm{JF}$, et al. Neurologic Complications of Infective Endocarditis: Recent Findings. Current Infectious Disease Reports. 2017; 19: 41.

[26] García-Cabrera E, Fernández-Hidalgo N, Almirante B, IvanovaGeorgieva R, Noureddine M, Plata A, et al. Neurological complications of infective endocarditis: risk factors, outcome, and impact of cardiac surgery: a multicenter observational study. Circulation. 2013; 127: 2272-2284.

[27] Cunha BA, Jimada I, Chawla K. Intracranial complications of acute bacterial endocarditis. Surgical Neurology International. 2018; 9: 107.

[28] Haysom L, Cross M, Anastasas R, Moore E, Hampton S. Prevalence and Risk Factors for Methicillin-Resistant Staphylococcus aureus (MRSA) Infections in Custodial Populations: a Systematic Review. Journal of Correctional Health Care. 2018; 24: 197-213.

[29] Veronese C, Pellegrini M, Maiolo C, Morara M, Armstrong GW, Ciardella AP. Multimodal ophthalmic imaging of staphylococcus aureus bacteremia associated with chorioretinitis, endocarditis, and multifocal brain abscesses. American Journal of Ophthalmology Case Reports. 2020; 17: 100577.

[30] Mehmood MA, Patel M, Sanekommu H. Methicillin-Resistant Staphylococcus Aureus: a very Rare Cause of Meningitis. Cureus. 2020; 12: e10370

[31] Pintado V, Pazos R, Jiménez-Mejías ME, Rodríguez-Guardado A, Gil A, García-Lechuz JM, et al. Methicillin-resistant Staphylococcus aureus meningitis in adults: a multicenter study of 86 cases. Medicine. 2012; 91: 10-17.

[32] Nair AG; Rathi N, Apte MK, Marathe TR, Potdar NA, Shinde CA. Simultaneous Bilateral Orbital Cellulitis with Meningitis Caused by Methicillin-Resistant Staphylococcus aureus in an Immunocompetent Infant. Journal of Pediatric Ophthalmology \& Strabismus. 2020; 57: e34-e37.

[33] Yagi S, Takahashi T, Murakami K, Azuma M, Sugano M, Miyamoto R, et al. Infective Endocarditis from Furuncle with Meningitis Complication Caused by Methicillin-resistant Staphylococcus aureus. Internal Medicine 2021; 60: 32513255.

[34] Longhurst WD, Sheele JM. Spontaneous methicillin-resistant Staphylococcus aureus (MRSA) meningitis. The American Journal of Emergency Medicine. 2018; 36: 909.e1-909.e3.

[35] Jia Y, Wang W, Wang X, Jiao L, Wang Y. Staphylococcus aureus meningitis complicated with intracranial hemorrhage and cerebral infarction: a case report. International Journal of Neuroscience. $2021 ; 31: 1-4$

[36] Oshima N, Hamada H, Hirose S, Shimoyama K, Fujimori M, Honda $\mathrm{T}$, et al. Panton-Valentine leukocidin-positive novel sequence type 5959 community-acquired methicillin-resistant Staphylococcus aureus meningitis complicated by cerebral infarction in a 1-month-old infant. Journal of Infection and Chemotherapy. 2021; 27: 103-106

[37] De Schryver N, Cosnard G, van Pesch V, Godfraind C, Hantson P. Extensive Spinal Cord Injury following Staphylococcus au- reus Septicemia and Meningitis. Case Reports in Neurology. 2011; 3: 147-153.

[38] Kumari A, Agrawal SC. Pneumocephalus consequent to staphylococcal pneumonia and meningitis. Journal of Pediatric Neurosciences. 2011; 6: 84-86.

[39] Aijazi I, Abdulla FM, Ibrahim AE. COMMUNITY ACQUIRED METHICILLIN SENSITIVE STAPHYLOCCUS AUREUS CEREBRAL ABSCESS IN A PREVIOUSLY HEALTH GENTLEMAN MIMICKING SIGNS OF CAVERNOUS SINUS THROMBOSIS-A UNIQUE PRESENTATION. Journal of Ayub Medical Collection Abbottabad. 2015; 27: 245-247.

[40] Kino H, Suzuki H, Yamaguchi T, Notake S, Oishi T, Ito Y, et al. Central nervous system infection caused by vancomycinintermediate Staphylococcus aureus (SCCmec type IV, ST8). Journal of Infection and Chemotherapy. 2014; 20: 643-646.

[41] Liu J, Bai R, Li Y, Staedtke V, Zhang S, van Zijl PCM, et $a l$. MRI detection of bacterial brain abscesses and monitoring of antibiotic treatment using bacCEST. Magnetic Resonance in Medicine. 2018; 80: 662-671.

[42] Gan YK, Azmi AZ, Ghani SA, Samsudin A. A rare case of methicillin resistant Staphylococcus aureus (MRSA) cerebral abscess secondary to conjunctivitis. The Medical Journal of Malaysia. 2017; 72: 197-198.

[43] Shichijo K, Ogose T, Kubota M, Tomimoto A, Kondo R, Taniguchi T, et al. Recurrent Staphylococcus aureus abscess and fatal pneumococcal septicemia due to IRAK-4 deficiency. Pediatrics International. 2015; 57: 1166-1169.

[44] Okada N, Nishiyama T, Kurihara M, Nishimura Y, Nishimura Y, Ando Y, et al. A case of panspinal epidural abscess that presented with meningeal irritation. Acute Medicine \& Surgery. 2017; 4: 363-366.

[45] Marinelli L, Trompetto C, Cocito L. Diffusion magnetic resonance imaging diagnostic relevance in pyogenic ventriculitis with an atypical presentation: a case report. BMC Research Notes. 2014; 7: 149.

[46] Lesourd A, Magne N, Soares A, Lemaitre C, Taha M, Gueit I, et al. Primary bacterial ventriculitis in adults, an emergent diagnosis challenge: report of a meningoccal case and review of the literature. BMC Infectious Diseases. 2018; 18: 226.

[47] Gronthoud F, Hassan I, Newton P. Primary pyogenic ventriculitis caused by Neisseria meningitidis: case report and review of the literature. JMM Case Reports. 2017; 4: e005078.

[48] Karakonstantis S, Galani D, Maragou S, Koulouridi A, Kalemaki D, Lydakis C. A rare case of acute transverse myelitis associated with Staphylococcusaureus bacteremia and osteomyelitis. Spinal Cord Series and Cases. 2017; 3: 17029.

[49] Saini M, Prasad K, Ling LM, Tan K. Transverse myelitis due to Staphylococcus aureus may occur without contiguous spread. Spinal Cord. 2014; 52 Suppl 2: S1-S2.

[50] Beh SC, Greenberg BM, Frohman T, Frohman EM. Transverse myelitis. Neurologic Clinics. 2013; 31: 79-138.

[51] He H, Jin L, Ju M, Tu G, Luo Z. Acute transverse myelitis of the cervical spine secondary to psoas abscess. BMC Infectious Diseases. 2016; 16: 579.

[52] Howard-Jones AR, Britton PN, Webster R, Ayer J, Khatami A. Mild encephalitis/encephalopathy with reversible splenial lesion in association with Staphylococcus aureus bacteraemia. Journal of Paediatrics \& Child Health. 2021; 9.

[53] Younessi OJ, Walker DM, Ellis P, Dwyer DE. Fatal Staphylococcus aureus infective endocarditis: the dental implications. Oral Surgery, Oral Medicine, Oral Pathology, Oral Radiology, and Endodontics. 1998; 85: 168-172.

[54] Valian SK, Mahmoudi S, Pourakbari B, Banar M, Ashtiani MTH, Mamishi S. The Causative Organisms of Bacterial Meningitis and their Antimicrobial Resistance Profiles in Iranian Children in 2011-2016. Infectious Disorders - Drug Targets. 2020, 
20: 229-236.

[55] Chen F, Chen Y, Zhao X, Wang J. Community-acquired methicillin-resistant Staphylococcus aureus ST59 in a Chinese adult with meningitis: A case report from China. Infection and Drug Resistance. 2020; 13: 2011-2016.

[56] Banks JT, Bharara S, Tubbs RS, Wolff CL, Gillespie GY, Markert JM, et al. Polymerase Chain Reaction for the Rapid Detection of Cerebrospinal Fluid Shunt or Ventriculostomy Infections. Neurosurgery. 2005; 57: 1237-1243.

[57] Hou Y, Zhang X, Hou X, Wu R, Wang Y, He X, et al. Rapid pathogen identification using a novel microarray-based assay with purulent meningitis in cerebrospinal fluid. Scientific Reports. 2018; 8: 15965.

[58] Kawaguchi T, Sakurai K, Hara M, Muto M, Nakagawa M, Tohyama $\mathrm{J}$, et al. Clinico-radiological features of subarachnoid hyperintensity on diffusion-weighted images in patients with meningitis. Clinical Radiology. 2012; 67: 306-312.

[59] Xu X, Li B, Yang H, Du Y, Li Y, Wang W, et al. Can diffusionweighted imaging be used to differentiate brain abscess from other ring-enhancing brain lesions? a meta-analysis. Clinical Radiology. 2014; 69: 909-915.

[60] Lai PH, Ho JT, Chen WL, Hsu SS, Wang JS, Pan HB, et al. Brain abscess and necrotic brain tumor: discrimination with proton MR spectroscopy and diffusion-weighted imaging. AJNR. American Journal of Neuroradiology. 2002; 23: 1369-1377.

[61] Pham AH, Le TD, Chu HT, Le TA, Duong HD, Van Dong $\mathrm{H}$. Infected intradural dermoid cyst without dermal sinus tract mimicking brain abscess: a case report. International Journal of Surgery Case Reports. 2020; 72: 142-146.

[62] Narayanan M, Mookherjee S, Spector TB, White AA. MSSA brain abscess and pyomyositis presenting as brain tumour and DVT. British medical journal Case Reports. 2013: bcr2013009380.

[63] Pujol M, Peña C, Pallares R, Ayats J, Ariza J, Gudiol F. Risk factors for nosocomial bacteremia due to methicillin-resistant Staphylococcus aureus. European Journal of Clinical Microbiology \& Infectious Diseases. 1994; 13: 96-102.

[64] Nagpal G, Flaherty JP, Benzon HT. Diskitis, Osteomyelitis, Spinal Epidural Abscess, Meningitis, and Endocarditis Following Sacroiliac Joint Injection for the Treatment of Low-Back Pain in a Patient on Therapy for Hepatitis C Virus. Regional Anesthesia and Pain Medicine. 2017; 42: 517-520.

[65] Skally M, Finn C, O'Brien D, Fitzpatrick F, Burns K, McFadden $\mathrm{E}$, et al. Invasive MRSA infections in neurosurgical patients - a decade of progress. British Journal of Neurosurgery. 2017; 31: 374-378.

[66] Ceraudo M, Prior A, Balestrino A, Anania P, Camera M, Fiaschi $\mathrm{P}$, et al. Ultra-short antibiotic prophylaxis guided by preoperative microbiological nasal swabs in endoscopic endonasal skull base surgery. Acta Neurochirurgica. 2021; 163: 369-382.

[67] Masterson TM, Rodeheaver GT, Morgan RF, Edlich RF. Bacte- riologic evaluation of electric clippers for surgical hair removal. American Journal of Surgery. 1984; 148: 301-302.

[68] Pirie S. Hand washing and surgical hand antisepsis. Journal of Perioperative Practice. 2010; 20: 169-172.

[69] Lu C, Zhang Y, Chen M, Zhong P, Chen Y, Yu J, et al. Population Pharmacokinetics and Dosing Regimen Optimization of Meropenem in Cerebrospinal Fluid and Plasma in Patients with Meningitis after Neurosurgery. Antimicrobial Agents and Chemotherapy. 2016; 60: 6619-6625.

[70] Rebai L, Fitouhi N, Daghmouri MA, Bahri K. Linezolid for the treatment of postneurosurgical infection caused by methicillin-resistant Staphylococcus. Surgical Neurology International. 2019; 10: 215.

[71] Chen H, Yang C, Tsai M, Liao C, Lee C. Linezolid as salvage therapy for central nervous system infections due to methicillinresistant Staphylococcus aureus at two medical centers in Taiwan. Journal of Microbiology, Immunology and Infection. 2019; 53: 909-915.

[72] Frame PT, Watanakunakorn C, McLaurin RL, Khodadad G. Penetration of nafcillin, methicillin, and cefazolin into human brain tissue. Neurosurgery. 1983; 12: 142-147.

[73] Weis S, Kesselmeier M, Davis JS, Morris AM, Lee S, Scherag A, et al. Cefazolin versus anti-staphylococcal penicillins for the treatment of patients with Staphylococcus aureus bacteraemia. Clinical Microbiology and Infection. 2019; 25: 818-827.

[74] Mermer S, Turhan T, Bolat E, Aydemir S, Yamazhan T, Pullukcu $\mathrm{H}$, et al. Ceftaroline versus vancomycin in the treatment of methicillin-resistant Staphylococcus aureus (MRSA) in an experimental MRSA meningitis model. Journal of Global Antimicrobial Resistance. 2020; 22: 147-151.

[75] Kuriakose SS, Rabbat M, Gallagher JC. Ceftaroline CSF concentrations in a patient with ventriculoperitoneal shunt-related meningitis. The Journal of Antimicrobial Chemotherapy. 2015; 70: 953-954.

[76] Paul M, Bishara J, Yahav D, Goldberg E, Neuberger A, GhanemZoubi $\mathrm{N}$, et al. Trimethoprim-sulfamethoxazole versus vancomycin for severe infections caused by meticillin resistant Staphylococcus aureus: randomised controlled trial. British Medical Journal. 2015; 350: h2219.

[77] Lee DH, Palermo B, Chowdhury M. Successful treatment of methicillin-resistant staphylococcus aureus meningitis with daptomycin. Clinical Infectious Diseases. 2008; 47: 588-590.

[78] Kelesidis T, Humphries R, Ward K, Lewinski MA, Yang OO. Combination therapy with daptomycin, linezolid, and rifampin as treatment option for MRSA meningitis and bacteremia. Diagnostic Microbiology and Infectious Disease. 2011; 71: 286-290.

[79] Nau R, Sörgel F, Eiffert H. Central nervous system infections and antimicrobial resistance: an evolving challenge. Current Opinion in Neurology. 2021; 34: 456-467.

[80] Lagunes L, Encina B, Ramirez-Estrada S. Current understanding in source control management in septic shock patients: a review. Annals of Translational Medicine. 2016; 4: 330. 